\title{
New freely available FORTRAN library for evaluating Sommerfeld integrals
}

George Y. Panasyuk, John C. Schotland, and Vadim A. Markel

Recent work focused on simplifying the computation of the electromagnetic Green's tensor may be of interest to researchers in the areas of near-field optics and nanophotonics.

Computing the electromagnetic fields produced by an aboveground radiating antenna has been one of the most basic problems in radioengineering for the past century. If the antenna in question is a dipole (i.e., it can be thought of as a pair of electric charges of equal magnitude but opposite sign, separated by some relatively small time-varying distance), the radiated fields are given by the so-called Green's tensor. Apart from the obvious applications in radiocommunications, the Green's tensor is also used in geophysical exploration of Earth and in near-field imaging and spectroscopy.

Unfortunately, the Green's tensor is not easy to compute even in the simple half-space geometry. A rather general analytical solution to the problem was given by Sommerfeld about 100 years ago. ${ }^{1}$ A contemporary exposition of the subject can be found in a paper by Maradudin and Mills. ${ }^{2}$ However, Sommerfeld's solution is expressed in terms of oscillatory integrals. (The term 'oscillatory' means that the integrated functions change sign or oscillate many times over the domain of integration.) To compute an oscillatory integral numerically, one must sample the integrand at a very large number of points. In some instances, this may become computationally inefficient. Evaluation of Sommerfeld integrals is confounded by the fact that the functions in question are not only oscillatory but also complex, singular (i.e., they diverge at some points in the complex plane), and are otherwise difficult to handle. Still, it may seem that, given the power of modern computers, numerical evaluation of the Sommerfeld integrals should not be a serious problem. However, this is not so. To quote from a paper by Jimenez, Cabrera, and Cuevas del Rio, ${ }^{3}$ who in 1996 wrote a (now apparently no longer
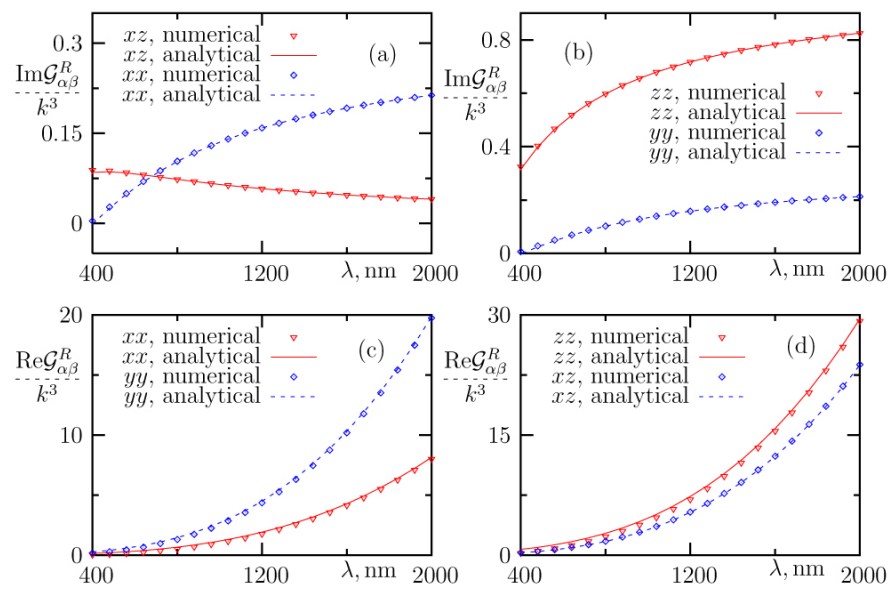

Figure 1. Various tensor components of the reflected ( $R$ ) part of the Green's tensor, $\mathcal{G}_{\alpha \beta}^{R}$, normalized by the free-space wave number $k=$ $\omega / c$, as functions of the free-space wavelength, $\lambda=2 \pi / k .(a, b)$ Imaginary $(\mathrm{Im})$ parts and $(c, d)$ real $(R e)$ parts of $\mathcal{G}^{R}$. The geometry is as follows: The source and the point of observation are located $40 \mathrm{~nm}$ above a transparent dielectric substrate and 40nm apart. The substrate dielectric permittivity is $\epsilon=2.5$ and assumed to be constant in the spectral range considered. The $x$-axis of the laboratory frame coincides with the line connecting the two points. The indices $\alpha$ and $\beta$ label the tensor components and can take the values $x, y$, or $z$. In this geometry, the tensor components $\mathcal{G}_{x y}^{R}$ and $\mathcal{G}_{y z}^{R}$ are identically zero. The results were obtained using the numerical integration capability of the GF package (centered symbols) and the analytical approximation, which is also implemented in the package (lines). The imaginary part of the Green's tensor is very accurately reproduced by the analytical approximationa result not obtainable from the electrostatic method of images.

available) FORTRAN library for computing the integrals: "Evaluating Sommerfeld integrals used to be a standard nightmare for many electromagnetic engineers due to the singular, oscillating, and divergent behavior of their integrands."

Continued on next page 
In any case, if the electromagnetic Green's tensor must be found in the half-space geometry, there is no other way but to confront the Sommerfeld integrals, and one would hope that a numerical package that handles all the difficulties mentioned above would be available by now. Yet, when faced with the problem ourselves, we could find neither a package nor a clearly defined analytical approximation appropriate for our problem! This has moved us to create such a package and to place it as a compressed file GF.tar.gz in a freely accessible depository. ${ }^{4}$ The package (referred to as GF below) can be also obtained in the supplementary material to our recent article in the Journal of Physics A. ${ }^{5}$ It is written in Fortran-77 and can be compiled with a freely available compiler, such as 977 . Apart from a purely numerical procedure, which is quite general, the GF package contains a set of novel approximations that are applicable in the near field.

We became interested in the Sommerfeld integrals because we wanted to know how the presence of an interface influences various radiative processes, such as spontaneous emission, in aggregates of molecules or nanoparticles placed on a substrate. The above is an important question in near-field sensing and spectroscopy, and to answer it, one needs to know the imaginary part of the Green's tensor with high precision. The simplest approximation that one can attempt to use is the electrostatic method of images. This approximation, however, fails to capture radiative effects. Indeed, radiation appears to be third order in the dimensionless parameter $\omega \mathcal{L} / c$, where $c$ is the speed of light in vacuum and $\mathcal{L}$ is a characteristic length of the problem. It can be, for example, the distance between two molecules placed above a substrate.

For the problem briefly stated above, it is desirable to know the Green's tensor as an analytical function of the frequency, $\omega$. Therefore, in addition to a purely numerical integrator, the GF package contains an implementation of a novel analytical approximation which we recently developed. ${ }^{5}$ In this approximation, the reflected part of the Green's tensor is expanded in powers of $\omega \mathcal{L} / c$. The zeroth-order term in this expansion gives the result otherwise obtainable by the electrostatic method of images. The first-order terms are identically zero, and the higher-order terms give corrections to the electrostatic limit. The radiative effects, however, appear only in the third order. Thus, if the half-space is nonabsorbing, the zero- and second-order terms are purely real. The nonvanishing imaginary part of the Green's tensor is obtained only when the third-order terms are accounted for. The expansion can be useful in a variety of physical problems when direct numerical integration is inadequate. Figure 1 illustrates its accuracy for a physical situation that is typical in near-field optics.
One interesting area to which the GF package can be applied is the study of surface plasmon polaritons (SPPs) in metal nanostructures placed above a substrate. SPPs are localized electronic excitations which can propagate along conducting and nonconducting interfaces. The SPPs can be confined to spatial areas much smaller than the wavelength, $\lambda$, at the carrying frequency. This feature favorably distinguishes SPPs from the modes of conventional optical fibers. In fact, SPPs can propagate not only along smooth interfaces but also in chains of metal nanoparticles. One potential application of such chains is nanowaveguides, which has attracted considerable recent attention $^{6-8}$ and was also of interest to us. ${ }^{9,10}$ The nanoparticle chains are typically placed on a substrate. However, accounting for this fact in theory or simulations proved to be difficult, as it requires knowledge of the half-space Green's tensor. The numerical integrator of the GF package is a useful tool for tackling this difficulty.

In summary, we have created a Fortran-77 package for evaluating the half-space electromagnetic Green's tensor. This GF package contains a numerical integrator of general applicability and an implementation of a novel analytical expansion that is applicable in the near field. The GF package is quite flexible and has many tunable input parameters, as well as a detailed user's guide. We would be glad to provide support to all potential academic users. In the near future, we plan to use the GF package in a study of SPP propagation in metal nanoparticle chains placed on a substrate.

\section{Author Information}

\section{George Y. Panasyuk and John C. Schotland}

Departments of Bioengineering and Electrical Engineering

University of Pennsylvania

Philadelphia, PA

http:/ / whale.seas.upenn.edu/gpanasyuk/

http:/ / www.seas.upenn.edu/be/dir/details/John_Schotland_ details.html

http:/ / www.seas.upenn.edu/be/

George Panasyuk is a research associate in the Department of Bioengineering. His expertise includes the inverse problems that arise in interpreting optical near-field images, radiative transport in strongly scattering media, and optical tomography. In the past, he has also contributed to the physics of liquid crystals, cold plasmas, and to condensed-matter theory.

Continued on next page 
John Schotland is a professor of bioengineering and electrical engineering. His research is focused on theoretical optical physics with applications to biomedical imaging and nanooptics. Areas of current interest include optical tomography and the optical imaging of nanoscale systems. Inverse problems, particularly those involving scattering, are a unifying theme that connects these areas.

\section{Vadim A. Markel}

Departments of Radiology and Bioengineering

University of Pennsylvania

Philadelphia, PA

http:/ / whale.seas.upenn.edu/vmarkel/

http:/ / whale.seas.upenn.edu

Vadim Markel is an assistant professor of radiology and bioengineering. He is a theoretical optical physicist whose main contributions are in the areas of scattering theory, optics of nanostructures, radiative transport, and associated inverse problems, primarily applied to biomedical imaging.

\section{References}

1. A. Sommerfeld, Über die Ausbreitung der Wellen in der drahtlosen Telegraphie, Ann. Phys. Leipz. 28 (4), pp. 665-736, 1909.

2. A. A. Maradudin and D. L. Mills, Scattering and absorption of electromagnetic radiation by semi-infinite medium in the presence of surface roughness, Phys. Rev. B 11, pp. 1392-1415, 1975.

3. E. Jimenez, F. J. Cabrera, and J. G. Cuevas del Rio, Sommerfeld: a FORTRAN library for computing Sommerfeld integrals, Antennas Propagat. Soc. Int'1 Symp. 2, pp. 966-969, 1996.

4. http:/ / whale.seas.upenn.edu/CODES/ GF Package (EM Green's function near an interface) written by G. Y. Panasyuk, J. C. Schotland, and V. A. Markel. Accessed 28 October 2009.

5. G. Y. Panasyuk, J. C. Schotland, and V. A. Markel, Short-distance expansion for the electromagnetic half-space Green's tensor: general results and an application to radiative lifetime calculations, J. Phys. A 42, p. 275203, 2009.

6. S. A. Maier, P. G. Kik, H. A. Atwater, S. Meltzer, E. Harel, B. E. Koel, and A. G Requicha, Local detection of electromagnetic energy transport below the diffraction limit in metal nanoparticle plasmon waveguide, Nat. Mater. 2, pp. 229-232, 2003.

7. A. Alu and N. Engheta, Theory of linear chains of metamaterial/plasmonic particles as subdiffraction optical nanotrasmission lines, Phys. Rev. B 74, p. 205436, 2006.

8. K. B. Crozier, E. Togan, E. Simsek, and T. Yang, Experimental measurement of the dispersion relations of the surface plasmon modes of metal nanoparticle chains, Opt. Express 15, pp. 17482-17493, 2007.

9. V. A. Markel and A. K. Sarychev, Propagation of surface plasmons in ordered and disordered chains of metal nanospheres, Phys. Rev. B 75, p. 085426, 2007.

10. A. A. Govyadinov and V. A. Markel, From slow to superluminal propagation: Dispersive properties of surface plasmon polaritons in linear chains of metallic nanospheroids, Phys. Rev. B 78, p. 035403, 2008. 